
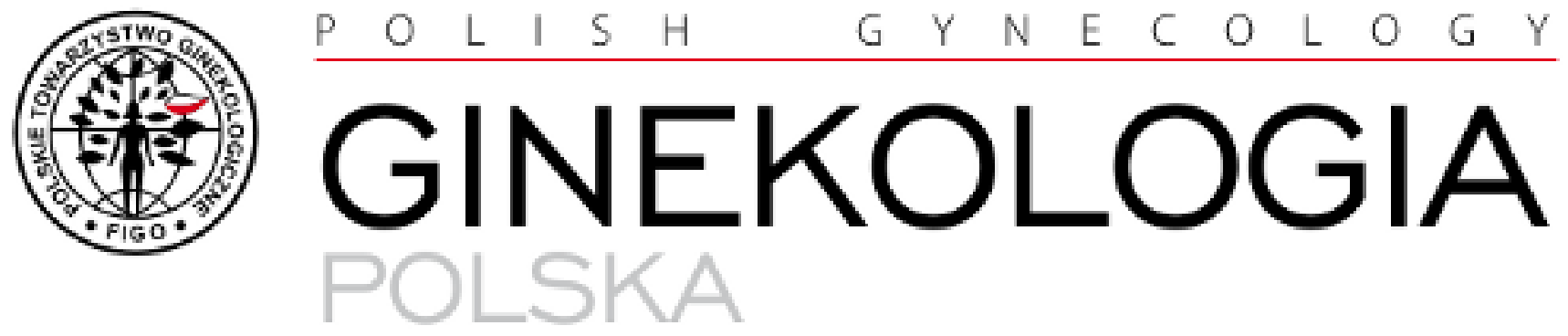

ORGAN POLSKIEGO TOWAAZYSTWA GINE KOLOGICZNEGO

THE OFFICIAL JOURNAL OF THE POLISH GYNECOLOGICAL SOCIETY

ISSN: 0017-0011

e-ISSN: $2543-6767$

\title{
Level of IGF1 in follicular fluid associated with IVF pregnancy outcome in the application of growth hormone
}

Authors: Luo Man, Yuanyuan Chen, Nannan Li, Yan Luo, Xiangyang Pan, Shaoming Zhou

DOI: $10.5603 / G P . a 2021.0232$

Article type: Research paper

Submitted: $2020-04-28$

Accepted: 2021-06-04

Published online: 2022-02-11

This article has been peer reviewed and published immediately upon acceptance.

It is an open access article, which means that it can be downloaded, printed, and distributed freely, provided the work is properly cited.

Articles in "Ginekologia Polska" are listed in PubMed. 


\title{
[ORIGINAL PAPER/GYNECOLOGY]
}

\section{Level of IGF1 in follicular fluid associated with IVF pregnancy outcome in the application of growth hormone}

Running title: IGF1 associated with IVF pregnancy outcome

Luo Man ${ }^{1}$, Yuanyuan Chen ${ }^{1}$, Nannan $\mathrm{Li}^{1}$, Yan Luo ${ }^{1}$, Xiangyang Pan ${ }^{1}$, Shaoming Zhou ${ }^{2}$ ${ }^{1}$ Department of Reproductive Center, The Maternal and Child Health Care Hospital of Hunan Province, Changsha, Hunan, China

${ }^{2}$ Department of Administration, The Maternal and Child Health Care Hospital of Hunan Province, Changsha, Hunan, China

\section{Corresponding author:}

Shaoming Zhou

Department of Administration, The Maternal and Child Health Care Hospital of Hunan Province, Changsha, Hunan, 410008, China

phone: 13973132132

e-mail: hunanivf@sina.com

\begin{abstract}
Objectives: The combination of growth hormone $(\mathrm{GH})$ with gonadotropin was a prevalent method to improve clinical reproduction in adjuvant for assisted reproduction treatment (ART). However, the contradictory results from previous studies failed to confirm the benefits. The present study is focused on the mechanism analysis of GH-IGF1-gonadal axis in ART and the changes of IGF1 in follicular fluid among different types of patients.
\end{abstract}

Material and methods: We recruited 136 patients and divided them into eight groups according to their ages and ovarian reserves. The baseline characteristics of the study population were summarized. The therapeutic outcomes in the study population were 
observed. In the meantime, concentrations of IGF1 in follicular fluids from different types of patients who underwent GH strategy were measured by Western blot. The functional mechanism of GH-IGF1-gonadal axis in ART was also analyzed.

Results: We analyzed the baseline characteristics of the study population, the therapeutic outcome of GH-IGF-1-gonadal axis, as well as the relative protein level of IGF1 and IGFBP1 in follicular fluid from different groups. The chemical pregnancy rate was significantly increased in different degrees for groups with GH co-treatment compared to groups without GH co-treatment. The IGF1 in follicular fluid of patients under 35 years' old showed an upward trend compared with groups of poor, normal and high ovarian reserves. After GH induction, IGF1 in follicular fluid was significantly increased in patients over 35 years old.

Conclusions: The study suggested that the application of GH might be beneficial to the pregnancy outcome in patients. GH application in patients older than 35 years might have a beneficial effect on pregnancy outcome via promoting the expression of IGF1. Our study indicates a different mechanism from GH application among younger and older patient in ART and provides a new clue for individual clinical treatment in infernity patients.

Key words: growth hormone, IGF-1, follicular fluid, in vitro fertilization

\section{INTRODUCTION}

In recent years, the incidence of female infertility among adult women has been rising all over the world [1]. The age-related fertility decline in the older women makes more and more families unable to achieve the desire of re-pregnancy and childbirth [2]. At present, assisted reproduction treatment (ART) based on in vitro fertilization (IVF) and embryo transfer (ET) has been considered as the most helpful method for female infertility. However, it can only ensure 1/3 of women get pregnant successfully, which cannot meet the urgent needs of the majority [3].

Previous studies have shown that patients with GH deficiency experienced reduced fertility rates based on IVF technology [4], while GH supplementation can successfully complete assisted reproduction [5]. Some researchers have indicated that co-treatment of gonadotropin and GH demonstrated significant improvement of the pregnancy, implantation and live birth rates in the patient with poor ovarian 
responders [6]. However, there is still great controversy in the application of GH in IVF, as well as the inconsistent meta-analysis in recent years [7, 8]. Further studies are urgently needed to gap the bridge between theoretical analysis and clinical application for the co-treatment of ART and GH.

GH-Insulin like growth factor 1 (IGF-1), also called somatomedin C, is a hormone with similar molecular structure to insulin, which plays an important role in pregnancy. It has been well established that IGF-1/gonadal axis exerts essential functions in follicular development, ovarian response and ovulation [9]. In the circulation, IGFs are bound to binding proteins (IGFBPs) that can prolong the halflife and modulate its bioavailability. In the ovary, IGFs are released by ovarian granulosa cells. Local IGFs exert function via IGF receptors to regulate normal follicular growth and development and raise the ovary sensitivity to the folliclestimulating hormone (FSH) [10]. Thus, the effects of GH on ovarian response may synergistically function as direct regulation and indirect stimulation of IGF1 synthesis.

Based on the potential synergistic effect of GH and IGF1 in IVF clinical output, we speculated that the difference in the clinical output of patients with different ages and ovarian reserves might be related to the concentration of IGF1 in ovaries. The identification of this speculation may provide valuable information for the explanation of the different clinical output of GH co-treatment strategies in ART. In this study, we were focused on the exploration of the role of GH-IGF1-gonadal axis in ART.

\section{MATERIAL AND METHODS}

\section{Study population}

The current research was performed in a single-blinded clinical trial, including 136 patients for IVF who referred to the Reproductive Center of The Hunan Maternal and Child Health Care Hospital, between June 2013 to June 2014. The patients were evaluated at the beginning and then assigned into eight groups. The study was approved by the Hunan Provincial Maternal and Children Health Hospital Ethics Committee. Written consents were obtained from the patients. The ethics approval number was provided (Identify Number: 2014005) by the ethics committee in 2014. The experiments were carried out according to guidance from Helsinki Declaration 
[11].

Patients were considered as eligible when they met the following criteria: [1] the causes of infertility are primarily due to fallopian tube malfunction or male sterility; [2] age between 20 and 45 years; [3] normal uterine cavity with regular spontaneous menstrual cycles of 25-30 days; and [4] FSH, Luteinizing hormone (LH), and Estradiol concentrations in the normal range during the early follicular phase. The exclusion criteria were as follows: [1] malignant tumor; [2] serious pelvic adhesions or hydrosalpinx; [3] endocrine disorder; [4] recurrent spontaneous abortion, [5] considering the side effect of $\mathrm{GH}$, patients who got positive results in OGTT were excluded, [6] patients who required fertilization by ICSI (single sperm microinjection) were excluded. In our study, we firstly recruited 136 patients. However, we found two women had high FSH levels (1 more than 20 IU/L, and 1 more than 15 IU/L), 4 were OGTT positive, and two gave up. They were excluded.

One hundred twenty-eight patients with ages less than 35 were classified into [1] poor, [2] normal and [3] high ovarian reserve groups according to their baseline FSH, FSH/LH ratio, anti-Müllerian hormone (AMH), and antral follicle count (AFC). For those patients, 36 patients who were identified as poor ovarian reserve were further divided into group A and group B randomly. Thirty-two patients who were identified as normal ovarian reserve were divided into group C and D randomly. Thirty patients who were identified as high ovarian reserve were set into groups $\mathrm{E}$ and F randomly. Thirty patients with ages over 35 were randomly categorized into groups $\mathrm{G}$ and $\mathrm{H}$. All the randomization work was done by a computerized random sampling table, with consideration on patient blindness. Finally, 15, 15, 15, 15, 18, 18, 16, 16 patients were distributed into group A, B, C, D, E, F, G and H, respectively.

\section{Study intervention}

The patients in all groups received a one-time gonadotropin-releasing hormone $(\mathrm{GnRH})$ agonist injection of triptorelin acetate (Diphereline, $3.75 \mathrm{mg} / \mathrm{bottle}$, Ipsen Pharma Biotech, France) for long-term pituitary down-regulation on day 15 of the preceding oral contraception pill cycle. GnRH-agonist dose ranged from $1.25 \mathrm{mg}$ to $1.875 \mathrm{mg}$ depending on the patients' body weights. On day $2-5$ of the next menstrual cycle, pituitary down-regulation was confirmed by an ultrasound scanned endometrial thickness (less than $5 \mathrm{~mm}$ ), as well as the serum FSH and LH levels (less than 5 
$\mathrm{mIU} / \mathrm{mL}$ ) and E2 level (less than $50 \mathrm{pg} / \mathrm{mL}$ ).

RFSH was given from the time when the downregulation is successful indicated in the above. The recovery of follicles was monitored. When the diameter of three or more follicles reached 7-9 mm, rFSH was given with doses varies from 150 to $300 \mathrm{IU}$ depending on individual ovarian responses and reserves. The rFSH dose was kept unchanged throughout the ovarian stimulation. If it is very necessary, the dose could be reduced by about $1 / 3$ in the last few days, and the reduction was maintained for 23 days each time. In addition to common regimens, groups B, D, F, H received 6 IU daily r-GH (Ansomon, Anke Co. LTD., Anhui, China) subcutaneously from the first day of gonadotropin (Gn) stimulation for 10 days. Group A, C, E, G received 10 days’ placebo (normal saline, $0.1 \mathrm{mg} /$ day) from the first day of Gn stimulation for 10 days, subcutaneously. $0.25 \mathrm{mg}$ Ovidrel (Merck Serono, Germany) was injected as the final trigger when dominant follicles reached $18 \mathrm{~mm}$ in diameter. Ultrasound-guided oocyte retrieval was performed at $36 \mathrm{~h}$ after the trigger.

Sixty mg per day of progesterone was started intramuscularly from the day of oocyte retrieval until 14 days after embryo transfer, together with $200 \mathrm{mg}$ oral progesterone capsules (Yimaxin, Xianju Pharmaceutical Co. LTD., Zhangjiang, China). Chemical pregnancy was confirmed with serum HCG $>40$ IU/L at day 14 after embryo transfer. Clinical pregnancy was confirmed with foteal heart activity that was observed under transvaginal ultrasonography at 4-5 weeks after embryo transfer and positive HCG indication. Progestin support continued up to 10-12 weeks' gestation if the pregnancy was achieved. After embryo transfer, we performed the measurement of total dosage and duration of gonadotropin usage, endometrial thickness, numbers of metaphase II oocyte, numbers of transferred embryos, and rates of early miscarriage, implantation, and clinical pregnancy.

\section{Assisted reproduction technique}

Oocytes were retrieved under vaginal ultrasonography guidance at 36 hours after r-HCG administrations and fertilized by traditional IVF procedures. During retrieval, $10 \mathrm{ml}$ follicular fluid was collected, frozen, and sent for analysis. Granulosa cell and corona radiata of cumulus oophorous were taken off. We assessed the maturity of the ova and found that the ova were naturally fertilized. After that, the zygotes were incubated for 18 hours in IVF nutrient solution at 37 Celsius with 5\% 
$\mathrm{CO}_{2}$. We observed the fertilization status at 24 hours and refreshed the nutrient solution. Embryo's evaluation was made on the $3^{\text {rd }}$ day after retrieval using Peter Score System. 1 or 2 embryos were transferred on the $3^{\text {rd }}$ day at cleavage stage to the uterine cavity.

Evaluations of embryos and zygotes were made by the standards previously reported by Tesarik et al. [12]. The morphology of the cleavage embryos was observed on the $2^{\text {nd }}$ and $3^{\text {rd }}$ day based on the number of fragmentations, equality, mono nuclearity, and early compaction. Patients in 8 groups were assessed in terms of collected oocytes, MII oocytes, fertilized oocytes, the number of transferred oocytes, and chemical or clinical pregnancy.

\section{Follicular fluid testing}

We measured the concentration of IGF1 in follicular fluid of different types of patients who underwent GH strategy and analyzed the functional mechanism of GHIGF1-gonadal axis in ART. Based on the randomized and double-blind principle, we collected the follicular fluid of the above eight groups. During retrieval, $10 \mathrm{~mL}$ follicular fluid was collected, frozen, and sent for analysis. Western blot was utilized to measure the levels of IGF1 and IGFBP1 in the follicular fluid of the patients. Image J (NIH software) was utilized to analyze the intensity of the western blot bands. Among the 128 patients, only 70 patients agreed to perform this test. Thus, 70 sets of western blot results were obtained for eight groups, with their number recorded in the experimental record sheet. Finally, there were 15, 13, 14, 12, 7, 2, 3, 4 samples in groups A, B, C, D, E, F, G, and H, respectively.

\section{Statistical analysis}

In this study, the data were expressed as mean \pm standard deviation (SD). The results were summarized utilizing absolute frequency and percentage for the categorical variable. Data normalization was performed utilizing Kolmogorov- 
Smirnoff tests. The differences among different groups were compared by one-way ANOVA or Kruskal-Wallis H test. SPSS (16.0) was utilized for statistical analysis. A p-value less than 0.05 was considered statistically significant.

\section{RESULTS}

One hundred twenty-eight patients were divided into eight groups according to their age and ovarian reserves. Table 1 showed the baseline characteristics of the study population. We investigated the mean age for female and male, BMI, number of IVF, level of AMH, antral follicle counts, and duration of infertility in the groups from A to $\mathrm{H}$. The classifications are in the following: group A represented patients with poor responses (age: < 35 years' old); group B represented patients with poor responses treated with growth hormone (age: < 35 years' old); group C represented patients with normal responses (age: < 35 years); group D represented patients with normal responses treated with growth hormone (age: < 35 years); group E represented patients with high responses (age: $<35$ years); group F represented patients with high responses treated with growth hormone (age: < 35 years); group G represented infertile women of advanced age (age: > 35 years); and group $\mathrm{H}$ represented infertile women of advanced age treated with growth hormone (age: > 35 years). From Table 1, we could find that the baseline characteristics of each group showed no significant difference ( $p>0.05$ ), including mean age, body mass index, the number of IVF procedure, the level of $\mathrm{AMH}, \mathrm{FSH}$, and $\mathrm{LH}$ hormones, antral follicle counts, and duration of infertility.

Then, we investigated the therapeutic outcome in the study population, including the number of collected oocytes, number of MII oocytes, number of fertilized oocytes, number of transferred embryos, chemical pregnancy percentage, IVF rate, cleavage rate, high-quality embryo rate, embryo implantation rate, and pregnancy rate. Table 2 revealed that the chemical pregnancy rate was significantly increased in different degrees for groups with GH co-treatment compared to groups without GH cotreatment. In patients with poor responses (age: < 35 years’ old), GH significantly improved the number of fertilized oocytes. However, no significant difference was observed in normal ovarian reverse group, including the number of collected follicles, the number of MII oocytes, fertilization rate, cleavage rate, the number of transfer embryos, high-quality embryo rate and implantation rate. In high response population, 
high-quality embryo rates and embryo implantation rates were significantly increased. For patients over 35 years' old, the chemical pregnancy rate has been improved, but no significant difference was observed in all other data (Tab. 2).

Based on the randomized and double-blind principle, we collected the follicular fluid of the above eight groups. Western blot was utilized to measure the levels of IGF1 and IGFBP1 in the follicular fluid of the patients (figure 1). As only 70 patients agreed to perform this test, we obtained 70 sets of western blot results for 8 groups. Figure 1 listed all the Western blot bands from 70 patients. Table 3 summarized the quantitative results after gray-scale analysis. The results demonstrated that the IGF1 in follicular fluid of patients under 35 years' old showed an upward trend compared with groups of poor, normal and high ovarian reserves. However, there is no significant difference among poor, normal and high ovarian reserves groups. The level of IGF1 in patients' follicular fluid over 35 years' old was significantly decreased compared with that of patients under 35 years' old. There was no significant difference in IGFBP1 among each group. After GH induction, IGF1 in follicular fluid was significantly increased in patients over 35 years old, but there was no significant changed in other groups.

\section{DISCUSSION}

Growth hormone has been applied to improve clinical reproduction in ART for more than 30 years [13, 14]. However, the contradictory result from different researchers failed to confirm the benefits in terms of live birth rates with the use of adjuvant GH. The differences in clinical outcomes from GH application among different researchers may be related to individual differences in the complexity of GH-Insulin like Growth Factor (IGF)-1-gonadal axis. The fundamental theories of GH co-treatment remain to be further elucidated.

Our study focused on the levels of follicular fluid from different types of patients. We found that the level of IGF1 showed a decreasing trend from high-response patients to poor-response patients. A significant downregulation was observed in the older patients over 35 years' old in contrast with patients less than 35 years' old. After GH stimulation, different susceptibility was revealed in different types of patients. GH seems to have no upregulation effect in the level of IGF1 in follicular fluid from patients below age 35. However, a significant increase in IGF1 was found in patients 
over age 35. It indicated that there existed different functional mechanisms underlying younger and older patients in ART. Our study gave a reasonable explanation for previous contradictory findings and provided new clues for individual clinical treatment in ART.

The human genetic deficiency and animal model shed light on the roles in follicular development, ovarian response, and ovulation. Both GHRH or GH mutations in humans lead to puberty delayed and fertility declined. GHR knockout mice showed a decrease in the number of healthy and growing antral or pre-ovulatory follicles [10], which demonstrated that GH is necessary for optimal follicular maturation and survival. Previous studies have established that both in vivo and in vitro administration of GH could increase ovarian weight, follicular size, and promote human oocyte retrieval and fertilization rate [10]. Furthermore, GH was reported to improve the endometrial receptivity by increasing endometrial blood flow and cytokines release [15]. As a result, it is reasonable to conclude that GH could advance clinical reproduction. In this study, we found that co-treatment with GH in poor ovarian reserves could improve the chemical pregnancy rate, the number of fertilized oocytes and IVF rate. For older patient aged over 35, the increase of chemical pregnancy rate was confirmed, which is consistent with previous studies [12-14]. There are still some deviations in experimental results, which may be due to different treatment schemes. The oocyte quality decline with age could be resulted from the fact that functional mitochondria decrease led to impaired separation of chromosome. GH can also improve the mitochondria activity other than promoting proliferation and inhibiting apoptosis [16]. This could explain why GH promote chemical pregnancy rate in older patient.

Except for direct role in the oocyte, GH indirectly induces ovarian granulosa and thecal cells release IGF1, which raises the ovary sensitivity to gonadotropin [15]. IGF1 locally exerts the role in resuming meiosis of the oocytes via paracrine and autocrine modes, including DNA synthesis, steroidogenesis, aromatase activity, LH receptor synthesis, and inhibin secretion [17]. In synergy with FSH, IGF1 is considered to mediate growth-promoting actions of growth hormone. IGF1 is required for GH to stimulate oocyte maturation. In our study, the level of IGF1 in follicular fluid showed a decreasing trend from high response patients to poor response patients. Significant downregulation of IGF1 was found in older patients. It indicated that the baseline of IGF1 might be related to ovarian response and clinical outcome. GH 
seems to have the effects of selective upregulation of IGF1 in older patients rather than younger patients. This may result from the different responses from normally and highly reactive patients. The high background level of IGF1 may saturate the GH effect. However, IGF1 cannot completely mediate the GH function. There might be an unknown mechanism underlying the resistance in the effect of GH for PORs. IGF1 knockout mice do not phenocopy the mutants with loss of GH and GHR. IGF1 cannot rescue the ovary deficiency caused by GHR mutants [18]. Loss of IGF1 in mice results in absence of antral follicles, infertility and fails to ovulate either spontaneously or under the influence of gonadotropins.

Different from GH, IGF1 plays a crucial role in the progression of the follicles from the non-gonadotropin sensitive to the gonadotropin sensitive stages [16]. It could be concluded that GH and IGF1 may synergistically function locally in follicular development. Our data show that the level of IGFBP1 in follicular fluid among each group did not change, suggesting that IGF1 is a local source rather than a circulation one. As IGF1 could bind with IGFBP1, lower IGF1 levels could increase the level of IGFBP1 in a certain time. In Table 3, we also found that IGF1 levels decreased, while IGFBP1 increased for group B compared with group A, and group D compared with group C. Follicular development is characterized by the proliferation and differentiation of the oocytes and granulosa cells. This process requires precise interaction between oocytes and granulosa cells. Through paracrine and autocrine ways, the balance of niche growth factors and cytokine steroids in follicles can be maintained. The good effect of granulosa cells on GH may help the older patients to achieve good clinical output in assisted reproduction.

Our study indicates a different mechanism underlying younger and older patients in ART and provides a new clue for individual clinical treatment in ART. However, as a limitation, our study did not identify the reason why GH did not regulate IGF1 in follicular fluid in POR patients but provided good clinical output. Further studies will be needed to investigate their inner associations and confirm the reasons and possible clinical outcomes. Due to space limitations and time constraints, this article did not include the plasma IGF1 experiments on this aspect. We will include the comparison between plasma IFG1 and IGF1 in the follicular fluid in the future research.

\section{CONCLUSIONS}


The study revealed that the chemical pregnancy rate was significantly increased in different degrees for groups with GH co-treatment compared to groups without GH co-treatment, suggesting that the application of $\mathrm{GH}$ may be beneficial to the pregnancy outcome in patients. The effects of GH in patients under 35 years of age might not be related to the expression of IGF1 and IGFBP1. GH application in patients older than 35 years might have a beneficial effect on pregnancy outcome via promoting the expression of IGF1. Our study indicates a different mechanism from GH application among younger and older patients in ART and provides a new clue for individual clinical treatment in ART.

\section{Ethics approval and consent to participate}

The study was approved by the Hunan Provincial Maternal and Children Health Hospital Ethics Committee. Written consents were obtained from the patients. The ethics approval number was provided (Identify Number: 2014005) by the ethics committee in 2014.

\section{Conflict of interest}

The authors declare that there is no conflict of interest.

\section{Consent for publication}

We confirm that the manuscript has been read and approved by all named authors and that there are no other persons who satisfied the criteria for authorship but are not listed. We further confirm that the order of authors listed in the manuscript has been approved by all of us.

\section{Data availability statement}

The authors confirm that all data underlying the findings are available. All relevant data are within the paper and its Supporting Information files.

\section{Author Contributions}

Luo Man performed the experiment and analyzed the data; Yuanyuan Chen, Nannan Li, Yan Luo and Xiangyang Pan performed the experiment; Shaoming Zhou guided the experiment, reviewed and edited the manuscript. 


\section{Acknowledgements}

The authors would like to thank The Maternal and Child Health Care Hospital of Hunan Province for their support.

\section{REFERENCES}

1. Fortin CS, Leader A, Mahutte N, et al. Gene expression analysis of follicular cells revealed inflammation as a potential IVF failure cause. J Assist Reprod Genet. 2019; 36(6): 1195-1210, doi: 10.1007/s10815-019-01447-4, indexed in Pubmed: $\underline{31001707}$.

2. García D, Brazal S, Rodríguez A, et al. Knowledge of age-related fertility decline in women: A systematic review. Eur J Obstet Gynecol Reprod Biol. 2018; 230: 109-118, doi: 10.1016/j.ejogrb.2018.09.030, indexed in Pubmed: 30248536.

3. Nørgård BM, Larsen MD, Friedman S, et al. Decreased chance of a live born child in women with rheumatoid arthritis after assisted reproduction treatment: a nationwide cohort study. Ann Rheum Dis. 2019; 78(3): 328-334, doi: 10.1136/annrheumdis-2018-214619, indexed in Pubmed: $\underline{30636215}$.

4. Aguiar-Oliveira MH, Bartke A. Growth Hormone Deficiency: Health and Longevity. Endocr Rev. 2019; 40(2): 575-601, doi: 10.1210/er.2018-00216, indexed in Pubmed: $\underline{30576428}$.

5. Correa FA, Bianchi PHM, Franca MM, et al. Successful pregnancies after adequate hormonal replacement in patients with combined pituitary hormone deficiencies. J Endocr Soc. 2017; 1(10): 1322-1330, doi: 10.1210/js.201700005, indexed in Pubmed: 29264457.

6. INVALID CITATION ! [12-14]. ??? (wrong reference???)

7. Norman RJ, Alvino H, Hull LM, et al. LIGHT investigators. Human growth hormone for poor responders: a randomized placebo-controlled trial provides 
no evidence for improved live birth rate. Reprod Biomed Online. 2019; 38(6): 908-915, doi: 10.1016/j.rbmo.2019.02.003, indexed in Pubmed: $\underline{30954433}$.

8. Dosouto C, Calaf J, Polo A, et al. Growth Hormone and Reproduction: Lessons Learned From Animal Models and Clinical Trials. Frontiers in Endocrinology. 2019; 10, doi: 10.3389/fendo.2019.00404.

9. Carter-Su C, Schwartz J, Argetsinger LS. Growth hormone signaling pathways. Growth Horm IGF Res. 2016; 28: 11-15, doi: 10.1016/j.ghir.2015.09.002, indexed in Pubmed: 26421979.

10. List EO, Sackmann-Sala L, Berryman DE, et al. Endocrine parameters and phenotypes of the growth hormone receptor gene disrupted (GHR-/-) mouse. Endocr Rev. 2011; 32(3): 356-386, doi: 10.1210/er.2010-0009, indexed in Pubmed: 21123740.

11. World Medical Association. World Medical Association Declaration of Helsinki: ethical principles for medical research involving human subjects. JAMA. 2013; 310(20): 2191-2194, doi: 10.1001/jama.2013.281053, indexed in Pubmed: 24141714.

12. Tesarik J, Greco E, Mendoza C. Late, but not early, paternal effect on human embryo development is related to sperm DNA fragmentation. Hum Reprod. 2004; 19(3): 611-615, doi: 10.1093/humrep/deh127, indexed in Pubmed: 14998960.

13. Carter-Su C. Growth hormone signal transduction. Growth Hormone \& IGF Research. 1998; 8(4): 311, doi: 10.1016/s1096-6374(98)80130-5.

14. Wondisford F. Growth Hormone. Essentials of Endocrinology and Metabolism. 2020: 229-240, doi: 10.1007/978-3-030-39572-8 26.

15. Cui N, Li AM, Luo ZY, et al. Effects of growth hormone on pregnancy rates of patients with thin endometrium. J Endocrinol Invest. 2019; 42(1): 27-35, doi: 10.1007/s40618-018-0877-1, indexed in Pubmed: 29671256.

16. Hrabia A, Sechman A, Gertler A, et al. Effect of growth hormone on steroid content, proliferation and apoptosis in the chicken ovary during sexual 
maturation. Cell Tissue Res. 2011; 345(1): 191-202, doi: 10.1007/s00441-0111187-5, indexed in Pubmed: 21618141.

17. Hull KL, Harvey S. Growth hormone and reproduction: a review of endocrine and autocrine/paracrine interactions. Int J Endocrinol. 2014; 2014: 234014, doi: 10.1155/2014/234014, indexed in Pubmed: 25580121.

18. European Society for Paediatric Endocrinology (ESPE). 2016, doi: 10.1159/isbn.978-3-318-05958-8.

Figure 1. Protein level of IGF1 and IGFBP1 in follicular fluid \#2, \#3, \#7, \#12, \#17, \#22, \#32, \#37, \#42, \#48, \#52, \#57, \#58, \#62, \#67 were from group A; \#8, \#13, \#18, \#23, \#27, \#28, \#33, \#38, \#43, \#47, \#53, \#63, \#68 were from group B; \#69, \#4, \#5, \#9, \#14, \#29, \#35, \#39, \#40, \#44, \#49, \#54, \#59, \#64 were from group C; \#10, \#15, \#19, \#20, \#24, \#34, \#45, \#50, \#55, \#60, \#65, \#70 were from group D; \#31, \#16, \#21, \#25, \#30, \#41, \#56 were from group E; \#51, \#66 were from group F; \#6, \#36, \#61 were from group G; \#1, \#11, \#26, \#46 were from group H

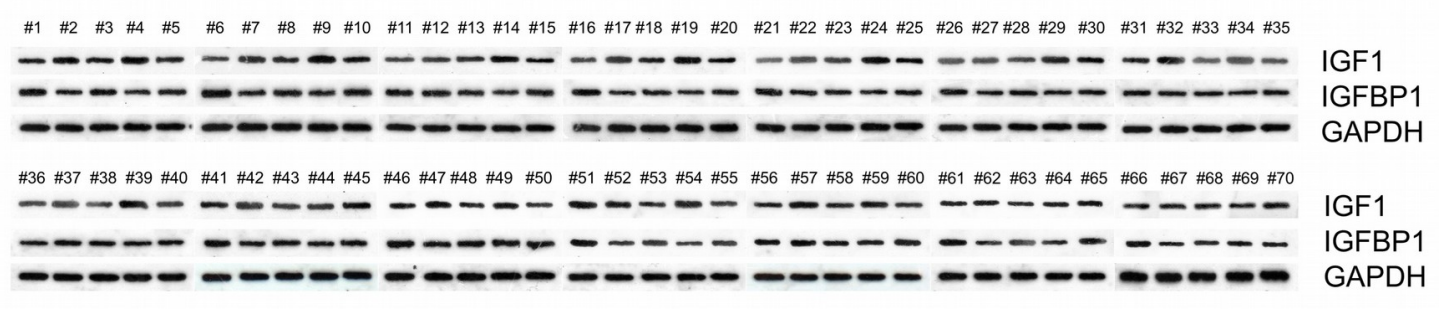

Table 1. Baseline Characteristics of the Study Population

There was no statistical significance between A and B, C and D, E and F, and G and H groups

\begin{tabular}{lllllllll}
\hline Index & Group & Group & Group & Group & Group & Group & Group & Group \\
& $\mathrm{A}$ & $\mathrm{B}$ & $\mathrm{C}$ & $\mathrm{D}$ & $\mathrm{E}$ & $\mathrm{F}$ & $\mathrm{G}$ & $\mathrm{H}$ \\
\hline Mean age, & 28.83 & 29.33 & 27.81 & 29.00 & 28.00 & 28.73 & 38.67 & $37.8 \pm$
\end{tabular}




\begin{tabular}{lllllllll}
\hline female & \pm 3.72 & \pm 2.19 & \pm 3.17 & \pm 2.63 & \pm 2.83 & \pm 3.07 & \pm 3.46 & 2.48 \\
Mean age, & 32.03 & 34.32 & 32.01 & 33.94 & 32.77 & 33.39 & 41.58 & 41.73 \\
male & \pm 5.28 & \pm 5.33 & \pm 4.47 & \pm 5.10 & \pm 4.87 & \pm 5.19 & \pm 2.97 & \pm 2.26 \\
BMI & 22.66 & 22.58 & 23.03 & 23.30 & 25.05 & 25.13 & 24.83 & 25.11 \\
{$[\mathrm{~kg} / \mathrm{m} 2]$} & \pm 2.87 & \pm 2.73 & \pm 3.17 & \pm 3.26 & \pm 5.34 & \pm 4.75 & \pm 2.22 & \pm 3.09 \\
Number of & $2.63 \pm$ & $2.28 \pm$ & $2 . .48 \pm$ & $2.57 \pm$ & $2.39 \pm$ & $2.56 \pm$ & $2.86 \pm$ & $2.93 \pm$ \\
IVF & 1.81 & 1.99 & 1.99 & 1.91 & 1.80 & 1.63 & 1.97 & 2.05 \\
Level of & $1.08 \pm$ & $0.97 \pm$ & $2.48 \pm$ & $2.73 \pm$ & $5.31 \pm$ & $6.03 \pm$ & $1.52 \pm$ & $1.48 \pm$ \\
AMH & 0.42 & 0.51 & $0.55^{*}$ & $0.64^{*}$ & $1.22^{*}, \#$ & $1.76^{*}, \#$ & 0.61 & 0.62 \\
Antral & $5.46 \pm$ & $7.37 \pm$ & 16.04 & 15.98 & 18.23 & 14.32 & $5.97 \pm$ & $8.18 \pm$ \\
follicle & 4.88 & 2.59 & $\pm 4.21^{*}$ & $\pm 5.13^{*}$ & $\pm 8.76^{*}$ & $\pm 5,16^{*}$ & 4.82 & 6.11 \\
counts & & & & & & & \\
Duration & $4.39 \pm$ & $4.67 \pm$ & $2.88 \pm$ & $4.07 \pm$ & $2.75 \pm$ & $2.47 \pm$ & $4.93 \pm$ & $6.07 \pm$ \\
of & 2.69 & 2.62 & 1.63 & 1.91 & 1.64 & 1.45 & 3.28 & 4.86 \\
infertility & & & & & & & \\
\hline
\end{tabular}

${ }^{*} \mathrm{p}<0.05$, vs A or B; ${ }^{\#} \mathrm{p}<0.05$, vs C or D. Group A: Patients with poor responses (age: $<35$ years' old); Group B: Patients with poor responses treated with growth hormone (age: < 35 years’ old); Group C: Patients with normal responses (age: < 35 years); Group D: Patients with normal responses treated with growth hormone (age: < 35 years); Group E: Patients with high responses (age: $<35$ years); Group F: Patients with high responses treated with growth hormone (age: < 35 years); Group G: infertile women of advanced age (age: > 35 years); and Group $\mathrm{H}$ : infertile women of advanced age treated with growth hormone (age: $>35$ years)

Table 2. The Therapeutic Outcome in the Study Population

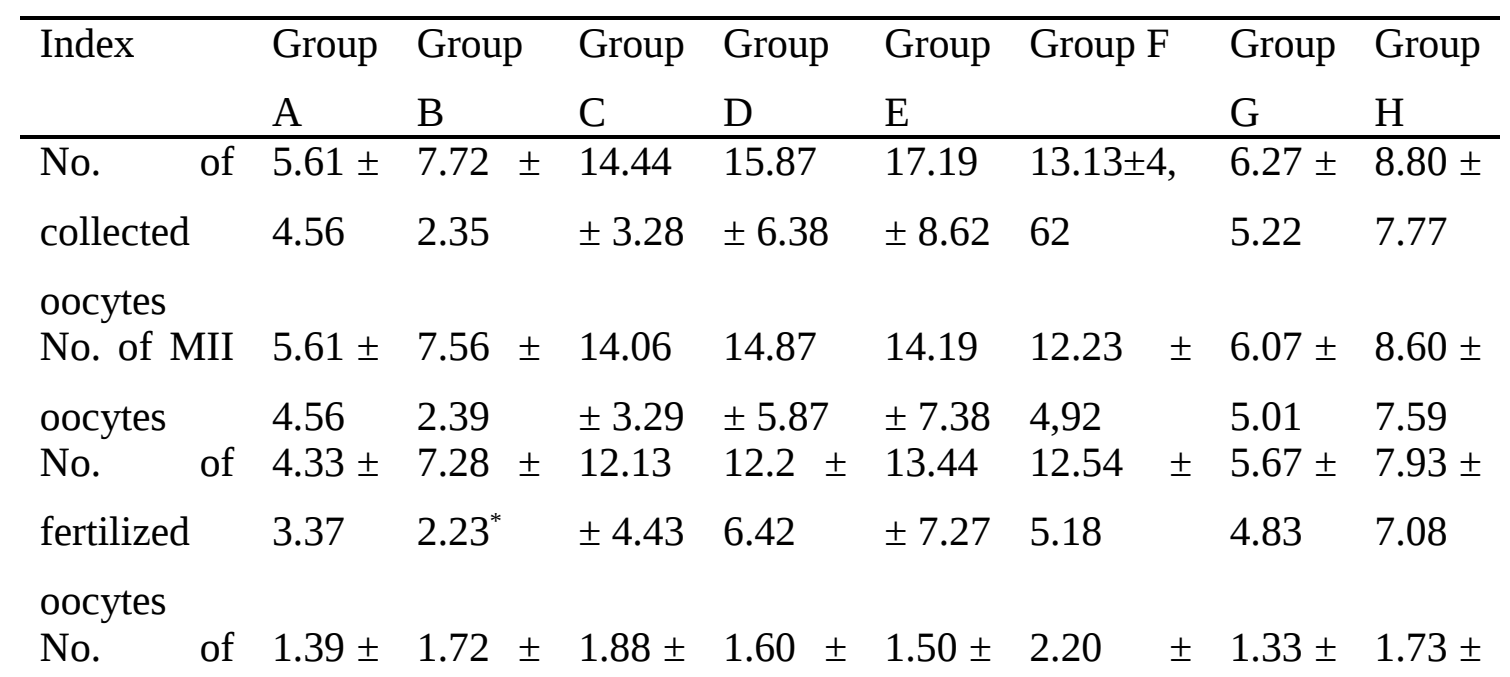




\begin{tabular}{|c|c|c|c|c|c|c|c|c|}
\hline transferred & 1.01 & 0.65 & 0.48 & 0.80 & 0.87 & $0.40^{*}$ & 1.14 & 1.18 \\
\hline $\begin{array}{l}\text { embryos } \\
\text { Chemical }\end{array}$ & $5(42$ & 11(69 & $7(46$ & 11(92 & $8(67$ & 12(80\%) & $7(33$ & $7(64$ \\
\hline pregnancy, & $\%)$ & $\%)$ & $\%)$ & $\%)$ & $\%)$ & & $\%)$ & $\%)$ \\
\hline$\%$ & & & & & & & & \\
\hline IVF rate & $0.95 \pm$ & $0.82 \pm$ & $0.84 \pm$ & $0.79 \pm$ & $0.84 \pm$ & 0.93 & $0.93 \pm$ & $0.91 \pm$ \\
\hline Cleavage & $\begin{array}{l}0.09 \\
0.94 \pm\end{array}$ & $\begin{array}{l}0.20^{*} \\
0.99 \pm\end{array}$ & $\begin{array}{l}0.22 \\
0.98 \pm\end{array}$ & $\begin{array}{l}0.31 \\
0.96 \pm\end{array}$ & $\begin{array}{l}0.26 \\
0.97 \pm\end{array}$ & $\begin{array}{l}0.12 \\
0.97\end{array}$ & $\begin{array}{l}0.12 \\
0.97 \pm\end{array}$ & $\begin{array}{l}0.15 \\
0.99 \pm\end{array}$ \\
\hline $\begin{array}{l}\text { rate } \\
\text { High }\end{array}$ & $\begin{array}{l}0.09 \\
0.51+\end{array}$ & $\begin{array}{l}0.03 \\
0.35+\end{array}$ & $\begin{array}{l}0.04 \\
0.42+\end{array}$ & $\begin{array}{l}0.05 \\
0.45+\end{array}$ & $\begin{array}{l}0.05 \\
0.29+\end{array}$ & $\begin{array}{l}0.06 \\
0.58\end{array}$ & $\begin{array}{l}0.08 \\
0.30+\end{array}$ & $\begin{array}{l}0.04 \\
0.22+\end{array}$ \\
\hline $\begin{array}{l}\text { quality } \\
\text { embryo }\end{array}$ & 0.30 & 0.31 & 0.28 & 0.25 & 0.26 & $0.32^{*}$ & 0.25 & 0.22 \\
\hline $\begin{array}{l}\text { rate } \\
\text { Embryo }\end{array}$ & $0.44 \pm$ & $0.21 \pm$ & $0.30 \pm$ & $0.54 \pm$ & $0.38 \pm$ & $0.63 \pm$ & $0.34 \pm$ & $0.29 \pm$ \\
\hline implantatio & 0.39 & 0.25 & 0.40 & 0.32 & 0.30 & $0.41^{*}$ & 0.64 & 0.36 \\
\hline $\begin{array}{l}\text { n rate } \\
\text { Pregnancy }\end{array}$ & $0.63 \pm$ & $0.42 \pm$ & $0.40 \pm$ & $0.83 \pm$ & $0.67 \pm$ & 0.80 & $0.33 \pm$ & $0.47 \pm$ \\
\hline rate & 0.48 & 0.49 & 0.49 & $0.37^{*}$ & 0.47 & 0.40 & 0.47 & 0.50 \\
\hline
\end{tabular}

${ }^{*} \mathrm{p}<0.05$ for Group A vs B; C vs D; E vs F; G vs H

Table 3. Relative protein level of IGF1 and IGFBP1 in follicular fluid

\begin{tabular}{lll}
\hline Index & IGF1/GAPDH & IGFBP1/GAPDH \\
\hline Group A & $0.503 \pm 0.062$ & $0.611 \pm 0.018$ \\
Group B & $0.480 \pm 0.067$ & $0.674 \pm 0.123$ \\
Group C & $0.526 \pm 0.022$ & $0.682 \pm 0.066$ \\
Group D & $0.493 \pm 0.054$ & $0.710 \pm 0.132$ \\
Group E & $0.557 \pm 0.091$ & $0.570 \pm 0.098$ \\
Group F & $0.573 \pm 0.114$ & $0.544 \pm 0.137$ \\
Group G & $0.474 \pm 0.073^{\#}$ & $0.579 \pm 0.087$ \\
Group H & $0.578 \pm 0.082^{*}$ & $0.544 \pm 0.118$ \\
\hline
\end{tabular}

${ }^{*} \mathrm{p}<0.05$ for Group H vs Group G, \#p $<0.05$ for Group E vs Group G 